\title{
Rotating stellar core-collapse waveform decomposition: a Principal Component Analysis approach
}

\author{
Ik Siong Heng \\ E-mail: i.heng@physics.gla.ac.uk
}

\begin{abstract}
.
This paper introduces the use of Principal Componenent Analysis as a method to decompose the catalogues of gravitational waveforms to produce a set of orthonormal basis vectors. We apply this method to a set of gravitational waveforms produced by rotating stellar core-collapse simulations and compare the basis vectors obtained with those obtained through Gram-Schmidt decomposition. We observe that, for the chosen set of waveforms, the performance of the two methods are comparable for minimal match requirements up to 0.9 , with 14 GramSchmidt basis vectors and 12 principal components required for a minimal match of 0.9. This implies that there are many common features in the chosen waveforms. Additionally, we observe the chosen waveforms have very similar features and a minimal match of 0.7 can be obtained by decomposing only waveforms generated from simulations with $\mathrm{A}=2$. We discuss the implications of this observation and the advantages of eigen-decomposing waveform catalogues with Principal Component Analysis.
\end{abstract}

\section{Introduction}

The current global network of interferometric detectors (GEO 600 [1], LIGO [2], TAMA 300 [3] and Virgo [4) have been scanning the sky for gravitational wave signals with unprecedented sensitivities. The prospect of detection is very real and once a detection is made, we must ask what can be inferred about the source from the detected gravitational wave signal. While this issue must be addressed for all signal types, we choose to focus on core-collapse supernovae here. Numerical relativity 
simulations have predicted several sets of gravitational wave signals or waveform catalogues due to rotating core-collapse supernovae (see [5] and references therein). The features of each waveform produced by the simulations vary depending on the physics employed and the chosen initial parameters. The predicted waveforms can be used as inputs into parameter estimation algorithms which can, for example, calculate the likelihood that the detected signal corresponds to one of the predicted waveforms. However, from a cursory inspection of the waveform catalogues, one can see that there are many common features in the predicted waveforms, especially for waveforms in the same catalogue. By decomposing the waveforms into a set of orthonormal basis vectors, we can greatly reduce the computation costs of the parameter estimation stage by concentrating on a subset of basis vectors that encompass the main features of the chosen waveforms.

We propose using Principal Component Analysis (PCA) to create an orthonormal set of basis vectors. Broadly speaking, PCA transforms a correlated, multi-dimensional data set into a set of orthogonal components. This is achieved by determining the eigenvectors and eigenvalues of the covariance matrix of the data set. The first principal component is the eigenvector with the largest corresponding eigenvalue. It is a the linear combination of the original variables which accounts for as much of the variability in the data as possible. Similarly, the second principal component is the linear combination which accounts for as much of the remaining variability as possible - subject to the constraint that it is orthogonal to the first principal component - and so on. In recent years, PCA has been applied to a number of astrophysical problems (see [6] for a recent example), such as spectral classification, photometric redshift determination and morphological analysis of galaxy redshift surveys, as well as wider class of image processing and pattern recognition problems across a range of scientific applications. For a detailed account of the statistical basis of PCA the reader is referred to, for example, Morrison (1967) [7] or Mardia et al. (1979) [8].

It must be noted that this is not the first approach proposed to decompose a waveform catalogue. Brady and Ray-Majumder [9] have previously applied Gram-Schmidt decomposition to the Zwerger-Müller [10] and Ott et al. [12] waveform catalogues. Additionally, Summerscales et al. [1] developed a Maximum Entropy based method to identify the presence of a gravitational wave signal and demonstrated the method's ability to extract the correct amplitude and phase of waveforms from a catalogue by Ott et al. (2004) [12]. 
In this article, we choose the waveform catalogue generated by simulations peformed by Dimmelmeier et al [13] to demonstrate the use of PCA. These simulations focus on rotating stellar core-collapse and the subsequent bounce. While this phase of a supernova has been the focus of numerous simulations over the years, recent simulations by Burrows et al. have produced large post-bounce gravitational wave signals due to acoustic shock mechanisms [14]. There is still much discussion about this mechanism so we choose to concentrate only on the gravitational wave signal produced by the core-collapse phase. Moreoever, we would like to stress that the choice of catalogue here is, to a large extent, arbitrary and the methods discussed in this paper can be easily applied to the other catalogues or any combination thereof. Additionally, we compare the basis vectors obtained by PCA and Gram-Schmidt decomposition by applying them to the same set of waveforms. We then describe our observations of the waveform catalogue before discussing our observations.

\section{Methods}

\subsection{Gram-Schmidt decomposition}

Gram-Schmidt (GS) decomposition is a recursive method for decomposing a set of waveforms to create a set of orthonormal basis vectors [15]. It was first applied to a supernova catalogue by Brady and Ray-Majumder [9]. For completeness, the main points of this method are reviewed below.

In GS decomposition, one begins by selecting a waveform from the data set as the first basis vector. To create a second basis vector, the first basis vector is first projected onto the next waveform to be included into the set of basis vectors. The projected component is then subtracted from the second waveform and the resulting vector is orthogonal to the first basis vector. One continues this process by subtracting the sum of the projections of all exisiting basis vectors onto the desired waveform. This is done recursively until the desired number of waveforms are included into the set of basis vectors. More explicitly, for a set of waveforms, $\left\{H_{1}, H_{2}, \ldots, H_{M}\right\}$, the orthonormal basis vectors, $\left\{e_{1}, e_{2}, \ldots, e_{M}\right\}$, are

$$
e_{i}=\bar{H}_{i} / \sqrt{\left(\bar{H}_{i}, \bar{H}_{i}\right)}
$$

with

$$
\bar{H}_{i}=H_{i}-\sum_{j=1}^{i-1}\left(H_{i}, e_{j}\right) e_{j}
$$


and $i=1, . ., M$ where $M$ is the total number of waveforms. Here, the brackets denote an inner product. Explicitly, the inner product for two vectors $a$ and $b$, each of length $n$ is given by

$$
(a, b)=\sum_{i=1}^{n} a_{i} b_{i}
$$

where $a_{i}$ denotes the $i^{\text {th }}$ element of the vector $a$.

Note that the second term in Equation 2 is the sum of the projection from all

previously formed basis vectors. Therefore, $\bar{H}_{i}$ is the residual waveform not described by previously generated basis vectors.

Brady and Majumder point out that the choice of the first waveform is chosen arbitrarily and may produce a basis vector set that spans the waveforms most efficiently. Therefore, the basis set is constructed repeatedly with a different initial waveform chosen each time until the basis vector that spans the waveforms' parameter with the fewest number of basis vectors is obtained.

\subsection{Principal Component Analysis}

In Principal Component Analysis (PCA), a basis set is formed by determining the eigenvectors of the covariance matrix of the desired data set. In the context of this article, let us arrange the waveforms from the catalogue $\{H\}$ into a matrix $\mathbf{H}$ such that each column corresponds to one of the waveforms, $H_{i}$. For $M$ waveforms, each of length $N$, the matrix $\mathbf{H}$ has dimensions of $N \times M$ and the covariance matrix for $\mathbf{H}$ is calculated by

$$
\mathbf{C}=\frac{1}{M} \mathbf{H H}^{\mathrm{T}} .
$$

where $\mathbf{C}$ is the covariance matrix with dimensions $N \times N$ for waveforms with length $N$. The normalised eigenvectors of $\mathbf{C}$ form a set of basis vectors, $\left\{e_{1}, e_{2}, \ldots, e_{M}\right\}$, that span the parameter space defined by the waveforms in $\mathbf{H}$. Note that in PCA, the eigenvalues of the covariance matrix tell us how well each eigenvector spans the parameter space of the waveform catalogue. The eigenvectors are, therefore, ranked by their corresponding eigenvalues, with the first principal component having the largest eigenvalue.

Supernovae waveforms have significant energies at high frequencies $(\sim 1 \mathrm{kHz})$, so $N$ can be about 1000 data samples at LIGO $(16384 \mathrm{~Hz})$ data sampling rates or more at Virgo $(20 \mathrm{kHz})$ sampling rates. Determining the eigenvectors of a matrix of 
such dimensions is computationally expensive. A common method of avoiding this computationally intensive operation (see [16] for example) is to first calculate the eigenvectors, $\mathbf{v}$, of $\mathbf{H}^{\mathrm{T}} \mathbf{H}$ such that

$$
\mathbf{H}^{\mathrm{T}} \mathbf{H} \mathbf{v}_{i}=\lambda_{i} \mathbf{v}_{i},
$$

where $\lambda_{i}$ is the corresponding eigenvalue for each eigenvector. Then, by premultiplying both sides by $\mathbf{H}$, we have

$$
\mathbf{H H}^{\mathrm{T}} \mathbf{H v}_{i}=\lambda_{i} \mathbf{H v}_{i} .
$$

If we rewrite Equation 4 so that the covariance matrix takes the form $\mathbf{C}=\mathbf{H H}^{T}$, then $\mathbf{H v}_{i}$ are the eigenvectors of the covariance matrix. $甘$ So, for $M \ll N$, we can determine the eigenvectors of covariance matrix by first calculating the eigenvectors of the smaller $\mathbf{H}^{T} \mathbf{H}$ which is an $M \times M$ matrix, thereby significantly reducing computation costs.

\section{Results}

\subsection{The waveforms}

The waveform catalogue used here to demonstrate the use of PCA were produced by Dimmelmeier et al [13. These waveforms are generated from axisymmetric general relativistic hydrodynamic simulations of stellar core-collapse to a proto-neutron star. They use the microphysics equation of state from Shen et al. [18] with a $20 M_{\odot}$ progenitor model from Woosley et al. [19]. There is a total of 54 waveforms in this catalogue generated by models which are parameterised by initial differential rotation, $A$, and the ratio of the rotational kinetic to gravitational energies, $\beta$. The values of $\beta$ are increased from $0.05 \%$ to $4 \%$ in 18 steps while three values of initial differential rotation were used. The three values, labelled $A=1, A=2$ and $A=3$, corresponding to differential rotation occurring at $50000 \mathrm{~km}$ (almost uniform rotation), $1000 \mathrm{~km}$ and $500 \mathrm{~km}$ respectively. According to the rotation law [20] the angular velocity has dropped to $1 / 2$ its central value at distance of $\mathrm{A}$ from the rotation axis. Hence, smaller values of A correspond to more differential rotation.

$\ddagger$ The method laid out here is similar to performing a singular value decomposition (SVD) [17 of the matrix $\mathbf{H}$. In SVD, equations 5 and 6 are the equivalent of using the right-singular vectors, which are the eigenvectors of $\mathbf{H}^{\mathrm{T}} \mathbf{H}$, to determine the left-singular vectors, which are the eigenvalues of $\mathbf{H H}^{\mathrm{T}}$. 


\subsection{Comparing GS and PCA basis vectors}

We introduce the match parameter, $\mu$, to quantify how well a set of basis vectors reconstructs a specified waveform. For a waveform, $H_{i}, \mu_{i}$ is calculated by summing the projections of the desired number of basis vectors, $Z$, onto the waveform such that

$$
\mu_{i}=\left\|\sum_{j=1}^{Z}\left(H_{i}, e_{j}\right) e_{j}\right\|
$$

where $e_{j}$ are the orthonormal basis vectors determined by the methods described in the previous section refbrady. As with equations 1 and 2 , the brackets denote an inner product. If we normalise the set of waveforms, then $\mu_{i}$ will be equal to 1 if the sum of the projections of the basis vectors match at particular waveform, $H_{i}$, exactly.

It is clear that $\mu$ will be equal to 1 for all waveforms in the catalogue if we use all basis vectors decomposed from the catalogue $(Z=M)$. However, it is interesting to calculate the smallest match obtained for any waveform in the catalogue (commonly referred to as minimal match) if we use a subset of basis vectors. The minimal match, $\mu_{\text {min }}$, is often used in templated matched filter searches for signals with wellmodelled waveforms. For such searches, the basis vectors form a bank of templates and minimal match is used to characterise how well the desired parameter space is covered by the template bank. To maximise the detection probability, one would maximise minimal match with the smallest number of templates so as to minimise computational time.

Computing time, however, is not a serious issue for these waveforms because they are short and relatively few compared to, for example, the number of templates used in a search for gravitational waves from binary neutron stars (see [21] for a recent example). Instead, we examine the minimal match here to study the parameter space covered by the waveform catalogue. If the parameter space of the waveform catalogue is degenerate, then one would expect the minimal match to rapidly approach 1 for $Z \ll M$.

Figure 1 shows the number of GS and PCA basis vectors required as a function minimal match. Similar number of GS and PCA basis vectors are required for minimal requirements up to about 0.9. The number of basis vectors required rises rapidly as the minimal match criterion approaches 1 since smaller features, unique to a small subset of waveforms, require a large number of basis vectors to reconstruct. 


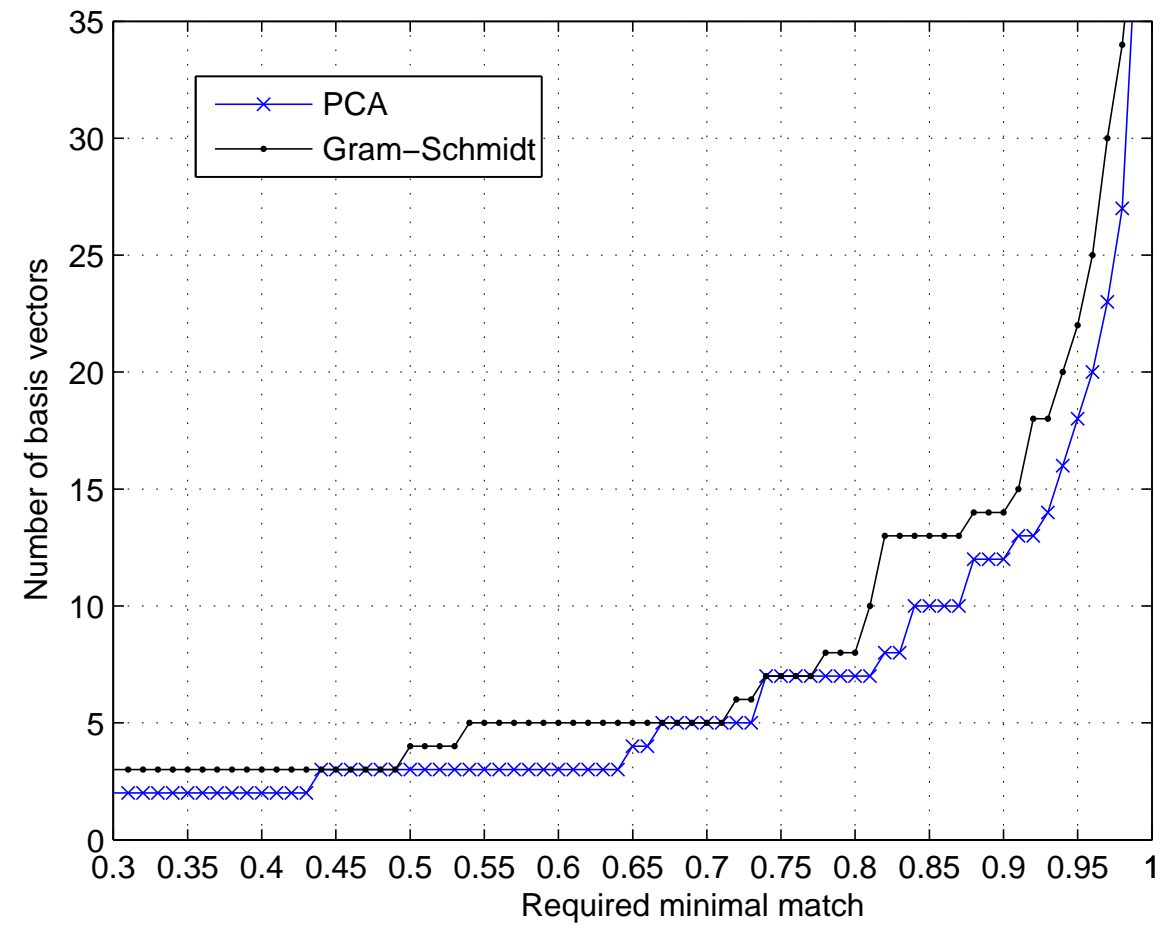

Figure 1. The number of PCA and GS basis vectors as a function of minimal match. For each value of minimal match, $\mu_{\min }$, we plot the number of basis vectors required so that $\mu>\mu_{\min }$ for all waveforms in the catalogue. The number of basis vectors required for each value of minimal match is comparable for both methods of decomposition.

It is interesting to note that for minimal match requirements greater than 0.95, more GS basis vectors are needed. Nonetheless, the parameter space spanned by the waveform catalogue is well spanned by less than half the total number of basis vectors for each method. This implies that all waveforms in the catalogue are dominated by a few unique features and this allows the minimal match to reach 0.75 with just 7 basis vectors. In fact, Dimmelmeier et al. noted that these waveforms can be divided into three broad categories: waveforms due to a pressure-dominated bounce with convective overturn, waveforms due to a pressure-dominated bounce only and waveforms with a single centrifugal bounce [13]. 
In Figure 2, we plot an example waveform reconstructed by the GS and PCA basis vectors with a match of 0.9 . The reconstructed waveform obtained by the two sets of basis vectors, though not identical, are very similar. The difference between the two waveforms is only about $10 \%$ in amplitude.
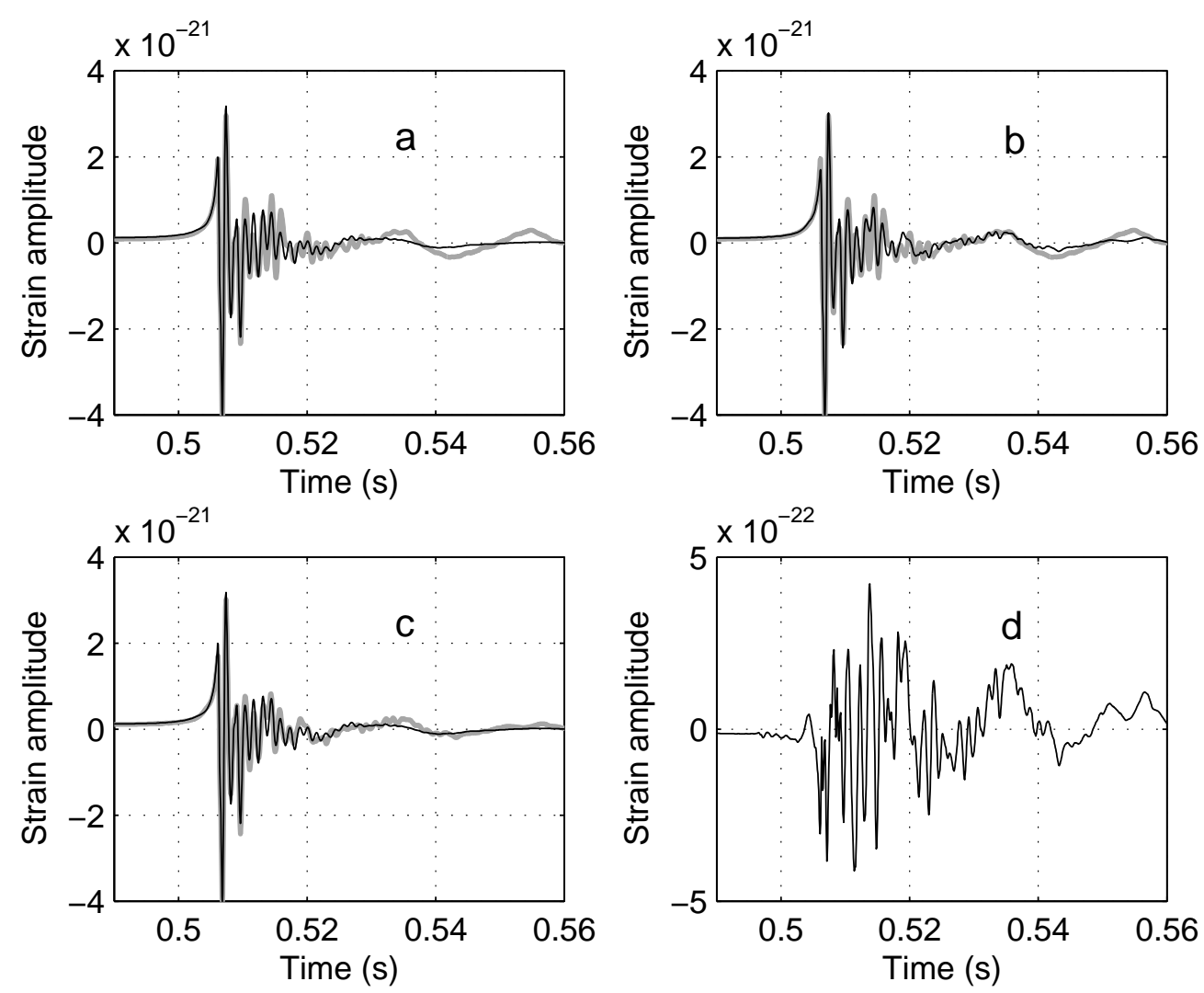

Figure 2. The reconstructed waveform using PCA and GS basis vectors are plotted as black line in figures (a) and (b) respectively. The grey line is the original waveform $(A=2, \beta=0.50)$ from the catalogue. A match of 0.95 was achieved using 6 PCA basis vectors and 17 GS basis vectors. For comparison, both reconstructed waveforms are plotted in (c), where the grey line is the PCA reconstruction and the GS reconstruction represented by the black line. The difference between the two reconstructions is plotted in $(\mathrm{d})$. 


\subsection{Using subset of the waveforms to form basis vectors}

In the previous subsection, we noted that the parameter space of the Dimmelmeier et $a l$. waveform catalogue used in our studies can be spanned by a small number of basis vectors. This implies that there are many common features in the waveforms from this catalogue. Here, we chose to make basis vectors using only the 18 waveforms with moderate differential rotation at $1000 \mathrm{~km}$ from the centre $(A=2)$. We make this choice to test the hypothesis that the waveforms from precollapse stellar cores with moderate differential rotation contain features presented in waveforms from low and highly differentially rotating stellar bodies. Figure 3 plots the number of waveforms with $A \neq 2$ observed to have match greater than 0.7 and 0.9 . With only 3 basis vectors, about 30 of the 36 waveforms already have a match greater than 0.7 . With $16 \mathrm{PCA}$ or GS basis vectors, $67 \%$ of the remaining 36 waveforms have match greater than 0.9 and $94 \%$ have match greater than 0.7. Therefore, a large fraction of the parameter space covered by the catalogue is covered by waveforms from simulations with $A=2$. This is consistent with the observations of Dimmelmeier et al [13, 22] who noted that the degree of differential rotation does not qualitatively alter the waveforms.

\section{Conclusions and discussion}

We have introduced PCA as a method of decompsing a set of waveforms into a set of basis vectors. A nice feature of PCA decomposition is that it allows one to quantitatively identify the main features in a desired set of waveforms since each basis vector is ranked by the value of its corresponding eigenvalue. One can interpret the basis vector with the largest corresponding eigenvalue (the first principal component) as having the most significant features in the waveform catalogue.

We compared the PCA method introduced here to the GS decomposition method introduced by Brady and Ray-Majumder. The efficiency of the PCA basis vectors at spanning the parameter space defined by the waveform catalogue are comparable to GS decomposition, with about 15 basis vectors required for a minimal match of 0.9 . For a minimal match of $0.95,17$ PCA basis vectors while 22 GS basis vectors were required. This shows that there are many common features in the waveforms from the chosen catalogue. We also generated a set of basis vectors using only the 18 of the 54 waveforms (with $A=2$ only) using both methods and observed that 34 


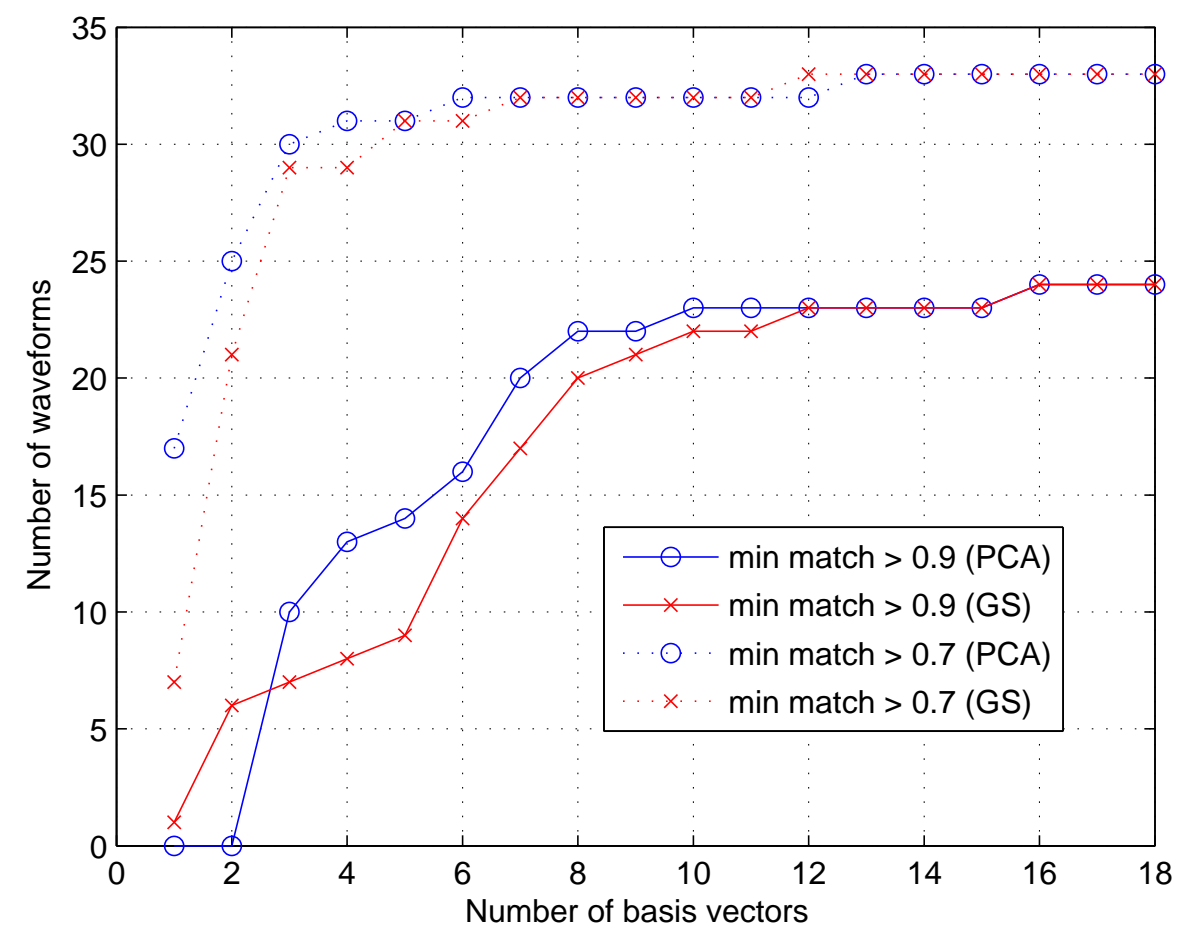

Figure 3. The number of waveforms with a match of at least 0.7 (dashed lines) and 0.9 (solid lines) as a function of the number of basis vectors.

of the 36 waveforms not included in the construction of the basis set have a minimal match of 0.7. This implies that the features from all waveforms are well described by models with $A=2$.

The basis vectors produced here can easily be used by parameter estimation techniques. For example, Monte-Carlo Markov-Chain (MCMC) methods [24] can be applied to a detected gravitational wave signal with each basis vector as a degree of freedom to search across. The output of the MCMC analysis would be a set of coefficients that can be used to reconstruct the signal waveform from a linear combination of basis vectors. Alternatively, we can project the waveforms onto the basis vectors to determine a set of coefficients with which we can reconstruct each waveform with the basis vectors. Each waveform can then be parameterised by these coefficients or weights and they can be used to form a classification scheme similar 
to that laid out by Turk and Pentland [23]. PCA as well as GS decomposition can also be used to decompose waveforms generated by simulations from different groups, using different core-collapse models. This application (also proposed by Brady and Ray-Majumder [9]) will combine the parameter space covered by all waveforms in an efficient manner. In the case of PCA, common features will be decomposed into the main components with large eigenvalues and, for parameter estimation, will reconstruct the main features of most waveforms. On the other hand, smaller features belonging to a small subset of waveforms will have much smaller eigenvalues and may be ignored by the analysis to reduce computation costs.

\section{Acknowledgments}

The author would like to thank Patrick Brady, Nelson Christensen, Harald Dimmelmeier, Martin Hendry, Christian Ott, Renate Meyer and Graham Woan for enlightening discussions. This work is supported by the Science and Technology Facilities Council and the Scottish Universities Physics Alliance.

\section{References}

[1] H. Lück et al., Class. Quantum Grav. 23 (2006) S71-S78.

[2] D. Sigg (for the LIGO Scientific Collaboration), Class. Quantum Grav. 23 (2006) S51-6.

[3] M. Ando and the TAMA Collaboration, Class. Quantum Grav. 22 (2005) S881-9.

[4] F. Acernese et al, Class. Quantum Grav. 23 (2006) S63-9.

[5] http://arxiv.org/abs/0809.0695

[6] M.J. Disney et al., Nature 455 (2008) 1082.

[7] D.F. Morrison 1967, Multivariate Statistical Methods, McGraw-Hill.

[8] K.V. Mardia, J.T. Kent and J.M. Bibby 1980, Multivariate Analysis (Probability and Mathematical Statistics), Academic Press.

[9] P.R. Brady and S. Ray-Majumder, Class. Quantum Grav. 21 (2004) S1839-47.

[10] T. Zwerger and E. Müller, Astron. Astrophys. 320 (1997) 209-227.

[11] T.Z. Summerscales, A. Burrows, L.S. Finn and C.D. Ott, Ap. J. 678 (2008) 1142-57.

[12] C.D. Ott, A. Burrows, E. Livne, and R. Walder, Ap. J., 600 (2004) 834.

[13] H. Dimmelmeier, C.D. Ott, H.-T. Janka, A. Marek and E. Müller Phys. Rev. Lett. 98 (2007) 251101.

[14] C.D. Ott, A. Burrows and Luc Dessart, Phys. Rev. Lett. 96 (2006) 201102.

[15] G. Golub and C. van Loan 1996, Matrix Computations, The John Hopkins University Press.

[16] R.A. Horn and C.R. Johnson 1990, Matrix Analysis, Cambridge University Press.

[17] G. Strang 1993, Introduction to Linear Algebra, Wellesley-Cambridge Press.

[18] H. Shen, H. Toki, K. Oyamatsu and K. Sumiyoshi, Prog. Theor. Phys. 100 (1999) 1013.

[19] S. E. Woosley, A. Heger and T. A. Weaver, Rev. Mod. Phys. 74 (2002) 1015.

[20] H. Komatsu, Y. Eriguchi and I. Hachisu, Mon. Not. R. astr. Soc. 237 (1989) 355-379. 
[21] B. Abbott et al., Phys. Rev. D 77 (2008) 062002.

[22] H. Dimmelmeier, C.D. Ott, A. Marek and H.-T. Janka, Phys. Rev. D 78 (2008) 064056.

[23] M. Turk and A. Pentland, Journal of Cognitive Neuroscience 3 (1991) 71-86

[24] W.R. Gilks, S. Richardson and D.J. Spiegelhalter 1996, Markov chain Monte Carlo in practice, Chapman \& Hall / CRC 

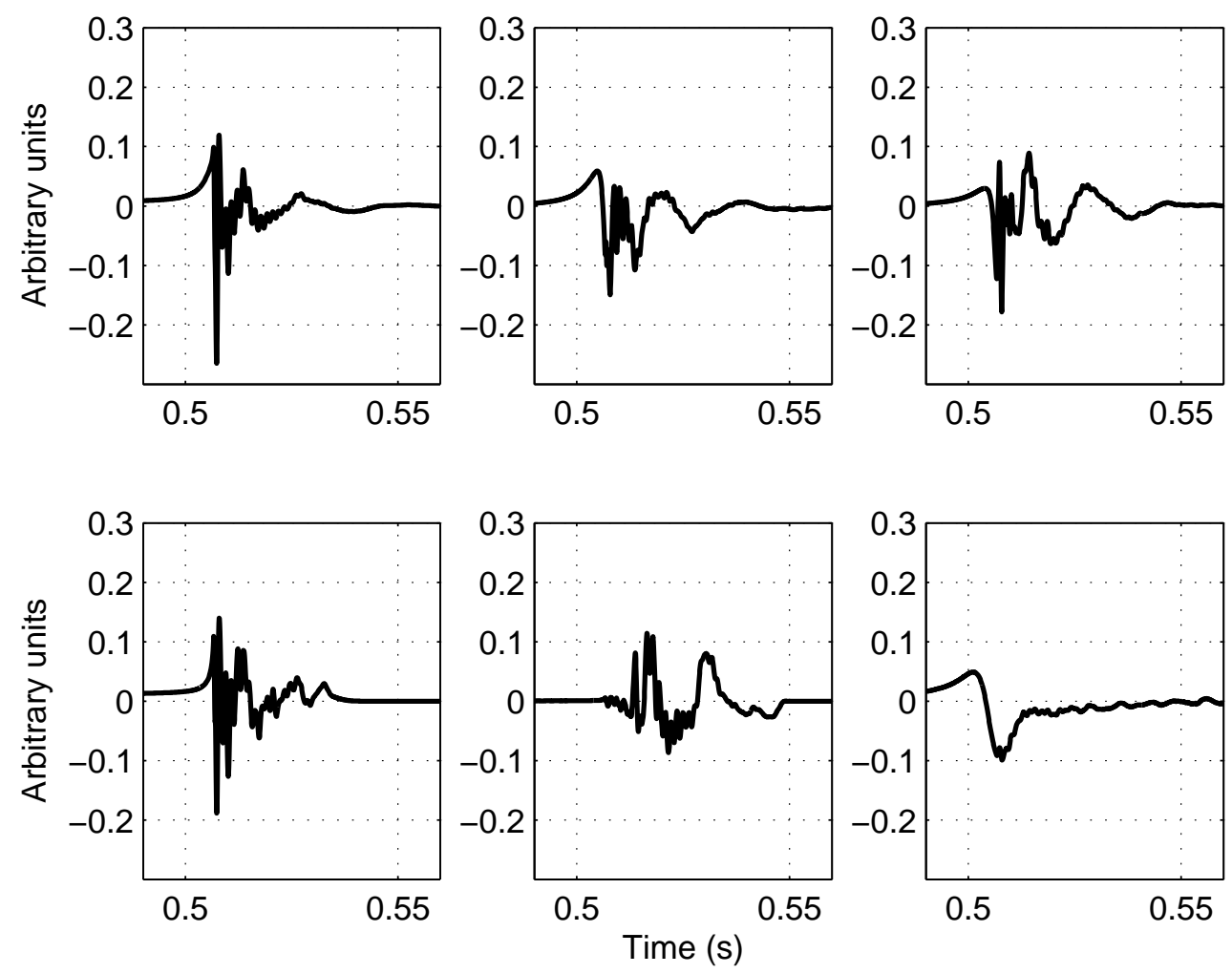

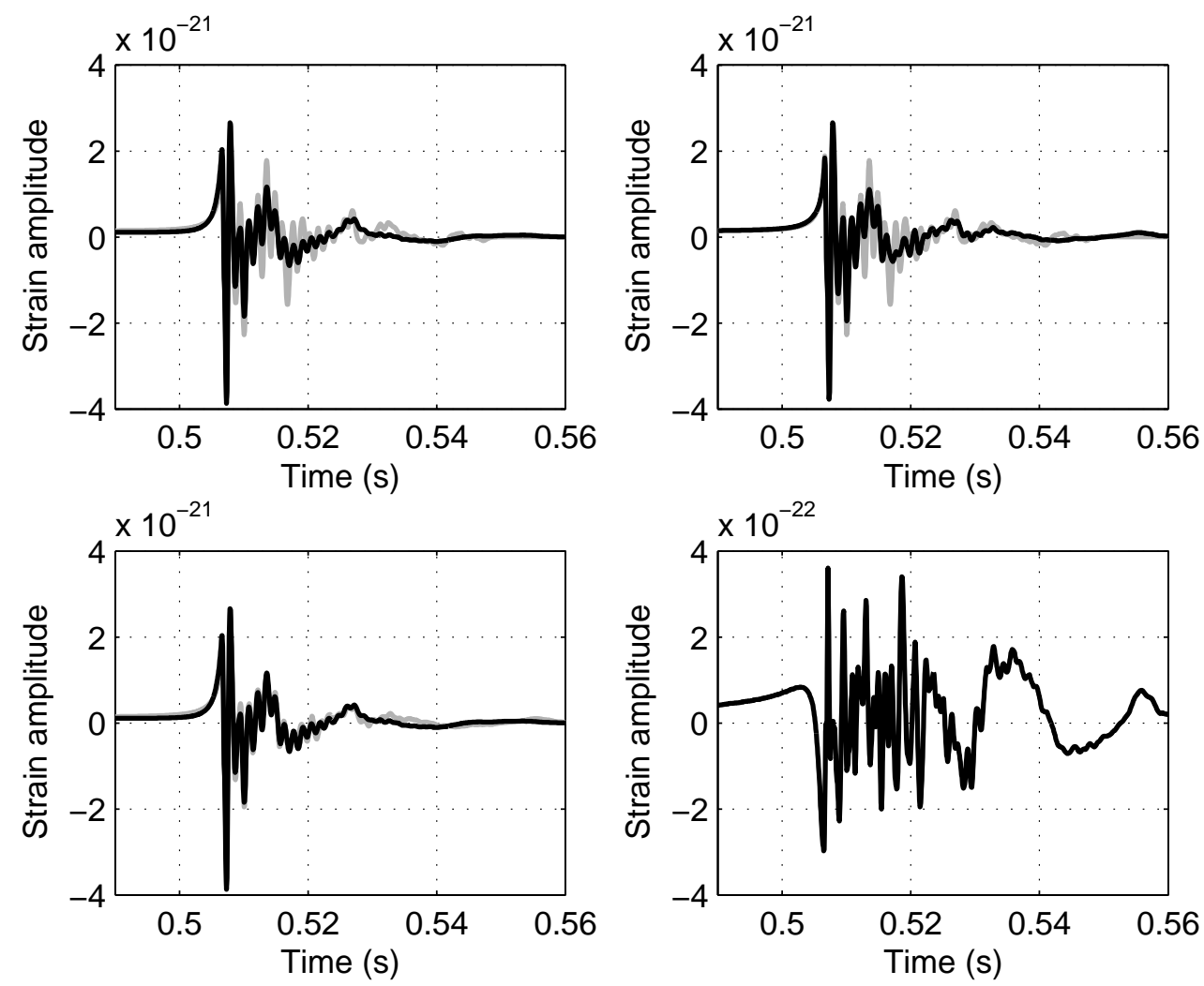

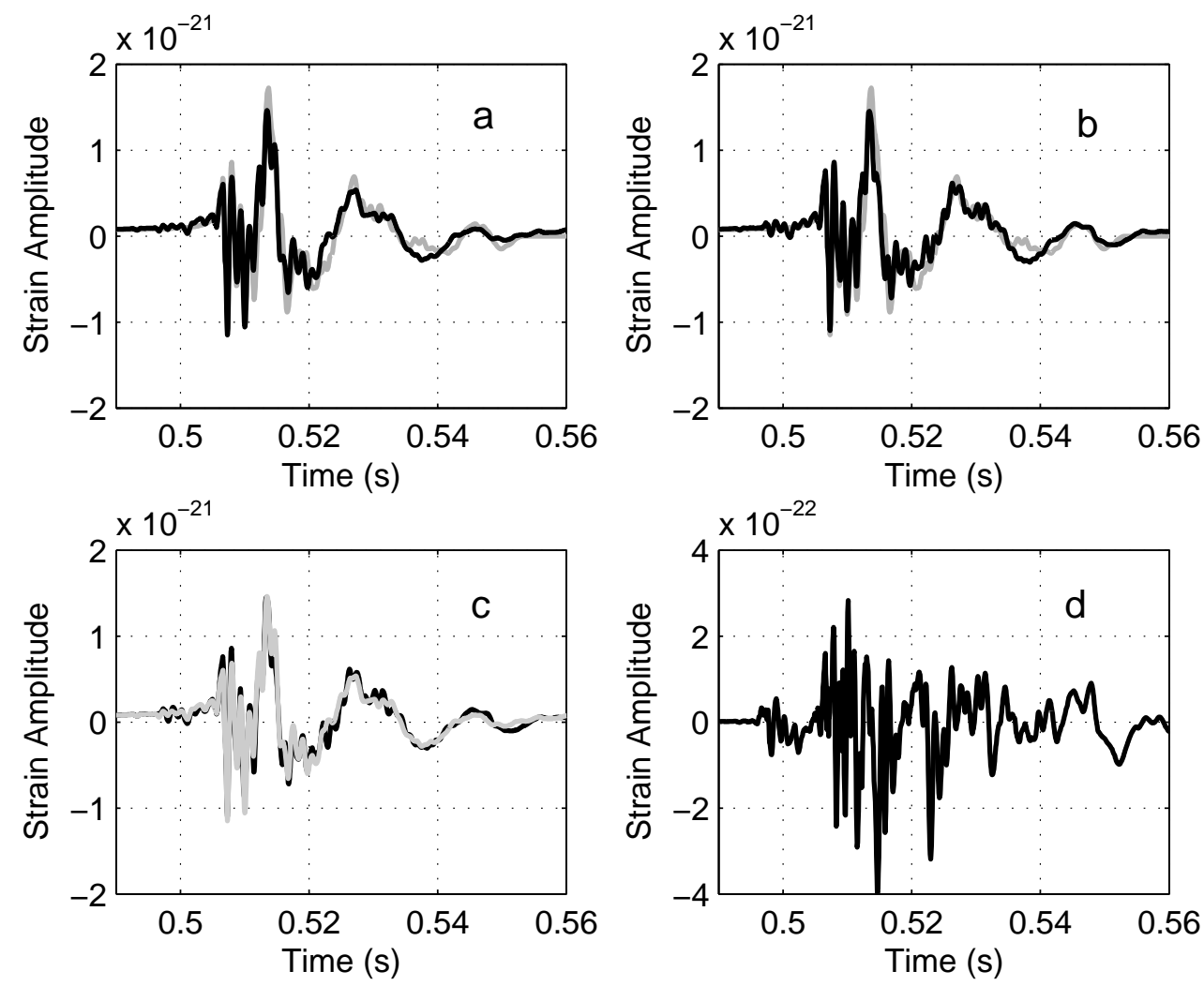

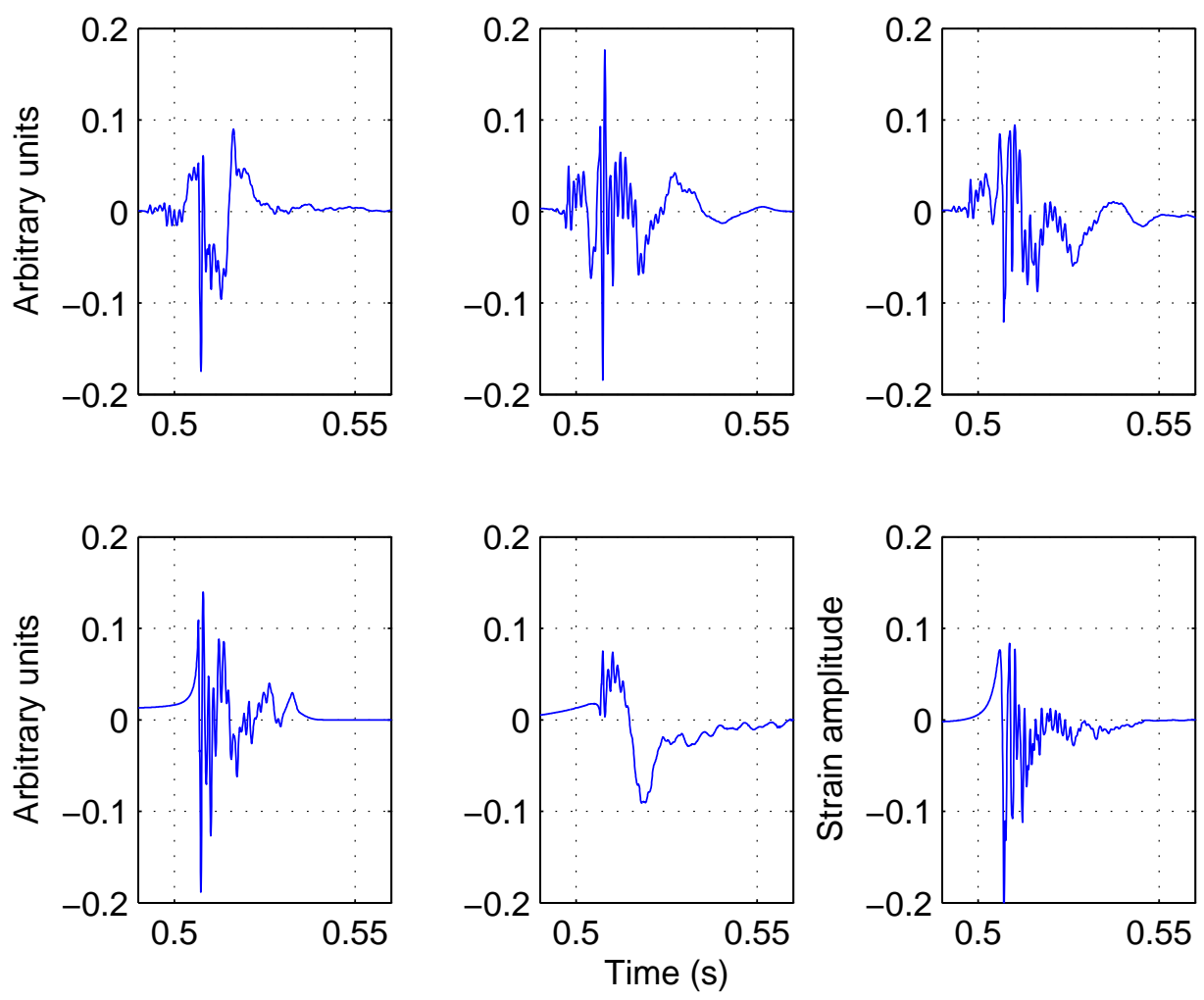
This figure "match_subset.png" is available in "png" format from: http://arxiv.org/ps/0810.5707v2 